\title{
Educación y salud intercultural: Reflexiones y miradas compartidas entre México y Brasil
}

\author{
Elizabeth Martínez Buenabad ${ }^{1}$ \\ Instituto de Ciencias Sociales y Humanidades "Alfonso Vélez Pliego" \\ Benemérita Universidad Autónoma de Puebla, México
}

Michele Neves Meneses ${ }^{2}$

Prefeitura Municipal do Rio Grande, Secretaria de Município da Saúde, Universidade Federal do Rio Grande do Sul-UFRGS, Porto Alegre, RS, Brasil

Resumen: La importancia de conformar equipos de investigación interdisciplinarios es enorme porque permite a sus integrantes trabajar con flexibilidad en el desarrollo académico, científico y sociocultural para facilitar el intercambio de información, conocimiento y experiencias de investigación con la finalidad de propiciar la reflexión conjunta a problemas afines. Así, bajo esta mecánica, las autoras del presente artículo pretendemos, proponer un diálogo profundo y analítico que recupere como eje central el tema de lo intercultural para dos campos básicos en toda sociedad: educación y salud. Para ello debatiremos en torno a temas como políticas públicas emanadas desde los Estados nacionales; del papel que juegan las instituciones y organizaciones encargadas de regular el tema de la educación superior y la salud (OMS, UNESCO, OPS) y, en contraparte, referiremos experiencias y metodologías autonómicas, desarrolladas en México y Brasil para generar prácticas y modelos alternativos que responden a las particularidades culturales de determinado grupo social.

Palabras clave: Educación; Salud; Interculturalidad; México; Brasil.

Título: Educação e Saúde Intercultural. Reflexões e olhares compartilhados entre México e Brasil

Resumo: A importância de criar equipes de pesquisa interdisciplinares é enorme, pois permite que seus membros trabalhem com flexibilidade no desenvolvimento acadêmico, científico e sociocultural para facilitar o intercâmbio de informação, conhecimento e experiências de pesquisa, com a finalidade de promover a reflexão conjunta de problemas afins. Assim, sob esse aspecto, as autoras do presente artigo pretendem propor um diálogo profundo e analítico que tenha como eixo central o tema da interculturalidade para dois campos básicos da sociedade: Educação e Saúde. Para isso, serão debatidos temas como políticas públicas advindas do Estado; do papel desempenhado pelas instituições e organizações responsáveis que direcionam o ensino superior e a saúde (OMS, UNESCO, OPAS) e, por outro lado, encaminhar-se-ão experiências e metodologias regionais, desenvolvidas no

\footnotetext{
${ }^{1}$ Doctora en Antropología por el Centro de Investigación y Estudios Superiores en Antropología Social (CIESAS), México. Profesora Investigadora del Instituto de Ciencias Sociales y Humanidades "Alfonso Vélez Pliego" de la Benemérita Universidad Autónoma de Puebla, México. Orcid: http://orcid.org/0000-0002-5026-6814.

E-mail: buenabad27@hotmail.com

${ }^{2}$ Mestra em Ensino na Saúde pela Universidade Federal do Rio Grande do Sul-UFRGS. Professora de educação em saúde. Superintendente de Vigilância em Saúde-Rio Grande/RS, Brasil. Orcid: https://orcid.org/0000-00020933-7051.
}

E-mail: michele.sms@riogrande.rs.gov.br 
México e no Brasil para estimular práticas e modelos alternativos que respondam às especificidades culturais de um determinado grupo social.

Palavras-chave: Educação; Saúde; Interculturalidade; México; Brasil.

Title: Education and intercultural health. Reflections and shared views between Mexico and Brazil

Abstract: The importance of forming interdisciplinary research teams is enormous because it allows its members to work flexibly in academic, scientific and sociocultural development to facilitate the exchange of information, knowledge and research experiences in order to promote joint reflection to related problems. Thus, under this methodology, the authors of this article intend to propose a deep and analytical dialogue that will recover as a central axis the issue of the intercultural for two basic fields in every society: education and health. In order to do so, we will discuss issues such as public policies emanating from national states; of the role played by institutions and organizations responsible for regulating the issue of higher education and health (WHO, UNESCO, PAHO) and, on the other hand, we will refer experiences and regional methodologies, developed in Mexico and Brazil to generate alternative practices and models that They respond to the cultural peculiarities of a certain social group.

Keywords: Education; Health; Interculturality; Mexico; Brazil.

\section{Introducción}

Es indiscutible que hoy en día enfrentamos el reto de responder con propuestas de educación y salud pertinentes, acorde a las demandas de los diferentes grupos sociales que conformamos el mundo, pero en particular esta América Latina y en donde debería primar el respeto a la diversidad de diversidades con sus múltiples problemas, pero también en donde se cuenta con un potencial de saberes y conocimientos ancestrales milenarios heredados de generación en generación y que, lamentablemente, siguen siendo negados o invisibilizados, no únicamente en las escuelas sino en la mayor parte de nuestras agencias estatales.

Es aquí donde el tema de la diversidad cobra relevancia en la época actual, siendo la universidad un espacio privilegiado para abordarlo, pero no sólo desde el punto de vista teórico, sino también desde la implicación que tiene en la vida cotidiana de las y los estudiantes, de las profesoras y profesores y en general, de todas y todos quienes cotidianamente construyen espacios de aprendizaje y posibilitan el diálogo sobre las distintas perspectivas que cada disciplina propone en las aulas desde las cuales será posible constituirse en un sujeto con la posibilidad de transformar su espacio y tiempo.

Esto nos motivó a conformar, desde el 2015, un equipo interdisciplinario de profesionales de la educación y salud de México y Brasil, en donde el objeto de estudio elegido para ser investigado en común fue la diversidad como parte de la vivencia y práctica cotidiana en espacios de formación y producción de conocimiento con la finalidad de generar propuestas transformadoras. 


\section{La diversidad sociocultural en Puebla y la formación universitaria intercultural}

Con un $\mathbf{1 7 . 6}$ por ciento de población indígena, Puebla es el cuarto estado con más habitantes indígenas en México, después de Oaxaca, Chiapas y Veracruz. En Puebla habitan un millón 18 mil 397 indígenas, lo que representa el 17.6\% de la población total del estado y el $9.96 \%$ de la población indígena a nivel nacional.

Pero además, es la tercera urbe con mayor población indígena. EI INSTITUTO NACIONAL DE ESTADÍSTICA GEOGRAFÍA E INFORMÁTICA (INEGI) (2015) ha señalado a Puebla como uno de los sitios que recibe flujos migratorios de indígenas con mayor frecuencia y magnitud, se trata por tanto de una población diversa compuesta por grupos de migrantes ya sean definitivos o de migración temporal.

Esto nos conduce a otra realidad no menos importante: ¿Cómo son abordadas estas realidades que implican una gama de diversidad sociocultural en las instituciones escolares, particularmente en las de educación superior? ¿Se ha reparado en los conocimientos nativos y lenguas maternas indígenas de los estudiantes o, por el contrario, simplemente son ignoradas? ¿Desde dónde y cómo pretendemos poner en marcha modelos educativos interculturales bilingües e inclusivos?

Advertimos que no estamos en contra de que nuestros estudiantes, sobre todo los de procedencia indígena, tengan que transitar hacia la adquisición y apropiación de conceptos, herramientas metodológicas y conocimientos occidentales, pero antes de ello tenemos que analizar y reorientar esa noción de educación para interculturalizar y ahora esa noción más reciente de una política de inclusión, la cual debería cuestionarse más como una tendencia institucional de pedagogización de la diversidad cultural vigente para asimilar y/o compensar, diferenciar y/o biculturalizar, tolerar y/o prevenir el racismo, transformar, interactuar, empoderar y descolonizar (DIETZ y MATEOS, 2011).

Sabemos de antemano que la escuela mexicana así ha formado a muchas generaciones, transmitiendo una sola cultura: la occidental y una sola lengua: el español. Esta herencia colonial ha sido muy difícil de transformar, prueba de ello es que sobre todo en licenciaturas en las que bien valdría aprovechar los intersaberes y los interaprendizajes, tanto de los estudiantes como de los docentes de procedencia indígena, no se ha piloteado todavía, nos referimos a los programas académicos como, derecho, medicina, enfermería, física, biología lingüística, por mencionar algunos.

Por ello, con el propósito de que la Benemérita Universidad Autónoma de Puebla cumpla a cabalidad la tarea de contribuir a la configuración de una "cultura científica" en la región, es imprescindible que la Universidad defina una política lingüística y cultural que tome en cuenta la naturaleza plurilingüe y pluricultural del país. La contribución al desarrollo de la cultura científica sólo puede darse a través de las lenguas nacionales: el castellano y las lenguas indígenas originarias, según dice el artículo 4 de la Ley General de Derechos Lingüísticos de los Pueblos Indígenas (DIARIO OFICIAL DE LA FEDERACIÓN, 2018). Desarrollar 
ciencia, básica y aplicada, en estas lenguas es imprescindible para consolidar la dimensión científica del Estado; sólo a partir de esta condición el país podrá entrar al intercambio mundial de conocimientos en una situación de menor desigualdad.

En ese sentido, es conveniente abrir la posibilidad de emplear las lenguas pertinentes en función de las actividades académicas que estén en el centro de interés. La lengua requerida depende de la disciplina, de la materia, del tema a explorar; de los contextos de uso (posgrado, licenciatura, preparatoria; de la editorial o revista), justamente es aquí en donde las instituciones de educación superior, en particular la BUAP, tienen mucho que hacer por delante entre sus académicos y estudiantes, pero también con un compromiso social, sustantivo, entre las presentes y futuras generaciones de mexicanos y en particular con la sociedad poblana.

Aquí, bien valdría reparar en lo que la UNESCO considera en los objetivos del nuevo milenio:

\begin{abstract}
La educación superior tiene la responsabilidad social de hacer avanzar nuestra comprensión de problemas polifacéticos con dimensiones sociales, económicas, científicas y culturales, así como nuestra capacidad de hacerles frente... por lo que debería centrarse aún más en los aspectos interdisciplinarios y promover el pensamiento crítico y la ciudadanía activa, contribuyendo así al desarrollo sostenible, la paz y el bienestar. (UNESCO, 2009, p. 2).
\end{abstract}

Por tanto, el tema de la educación intercultural enfrenta el gran desafío de romper viejas concepciones sobre quiénes son indígenas y de reconocer las historias negadas que configuran lo que es México. Este posicionamiento nos conduce a repensar la construcción de políticas públicas dirigidas, no únicamente a la población indígena, sino a la sociedad en su conjunto.

\title{
Las políticas y acciones internacionales en torno a la salud intercultural
}

Ahora bien, por lo que respecta al campo de la salud intercultural debemos remitirnos al Convenio 169 de la Organización Internacional del Trabajo (OIT, 2014), en su artículo 1 dice:

Los gobiernos deberán respetar la importancia especial que para las culturas y valores espirituales de los pueblos interesados reviste su relación con las tierras y territorios, o con ambos, según los casos que ocupan o utilizan de alguna otra manera y en particular los aspectos colectivos de esa relación (p. 10).

Si bien es cierto que, en materia de derechos humanos y salud contamos, como referente, con una Agenda de Salud para las Américas 2008-2017 y citamos: 
Una guía e inspiración para la formulación de políticas públicas y la ejecución de acciones para la salud, en pro del bienestar de la población de las Américas que reconoce que la Región es heterogénea y que nuestras naciones y sus poblaciones tienen necesidades distintas, y enfoques socioculturales diferentes para mejorar la salud (p. 5).

Esta Agenda se enmarca en el respeto y la adhesión a los siguientes principios y valores: Derechos humanos, universalidad, accesibilidad e inclusividad.

En esta misma dirección encontramos los Objetivos de desarrollo del milenio (2005): Una mirada desde América Latina y el Caribe que para su elaboración en el año 2000 convocó a una reunión de 147 países y 42 ministros. En la misma se aprecian una serie de metas, por ejemplo: como eje del desarrollo humano, la lucha contra la pobreza y el hambre, mejorar la salud materna, combatir el VIH/SIDA, el paludismo y otras. Pero una que destaca es la de Promover la capacidad de respuesta de los sistemas de salud reconociendo la diversidad cultural, por mencionar algunos.

Por otra parte, si revisamos el Artículo 12 del Pacto Internacional de Derechos Económicos, Sociales y Culturales, (2000) en relación con los pueblos indígenas, considera conveniente identificar los elementos que contribuirían a definir sus derechos a medidas específicas para mejorar su acceso a los servicios de salud y atención. Estos servicios de salud deben ser culturalmente apropiados, teniendo en cuenta los cuidados preventivos, las prácticas curativas y las medicinas:

Los Estados deben proporcionar recursos para los pueblos indígenas para diseñar, entregar y controlar este tipo de servicios para que puedan disfrutar del más alto nivel posible de salud física y mental. También deben protegerse las plantas medicinales, animales y minerales necesarios para el pleno disfrute de la salud de los pueblos indígenas también deben ser protegidos. (Párrafo 27).

Estos documentos sirvieron en el año del 2015 como referente para crear Los 17 Objetivos de Desarrollo Sostenible (ODS), también conocidos como Objetivos Mundiales (170 países) ONU (organización de las Naciones Unidas), son un llamado universal a la adopción de medidas para poner fin a la pobreza, proteger el planeta y garantizar que todas las personas gocen de paz y prosperidad. Entre estos objetivos destacamos el número 16: "Promover sociedades pacificas e inclusivas para el desarrollo sostenible, facilitar acceso a la justicia para todos y crear instituciones eficaces, responsables e inclusivas a todos los niveles" (ODS, 2015).

Sin embargo hoy en día poco se ha podido avanzar dentro de las instituciones de salud oficiales para el reconocimiento de los distintos sistemas de salud.

\section{La experiencia poblana: Los hospitales integrales}


En México la medicina tradicional se ha estudiado ampliamente y es, desde hace varias décadas, objeto de estudio privilegiado de la antropología. En apreciación de Fagetti (2003) "Se han investigado las enfermedades que cura y las creencias en las que se fundamenta, sin embargo, no se ha puesto el suficiente cuidado en evidenciar su eficacia" (párrafo 9). Los estudios de medicina tradicional han resaltado la importancia que ésta tiene para la población rural.

Se ha estudiado en tanto sistema médico que cura a los más pobres del país, pero no se ha tomado en cuenta que realmente cura y que puede convertirse en una alternativa para mucha gente, incluso para quienes viven en la ciudad. Está comprobado que curanderos y curanderas atienden a quienes padecen enfermedades denominadas síndromes de filiación cultural, que supuestamente sólo se conocen en los pueblos, y nada más.

No negamos que no se hayan buscado medidas encaminadas para ello, por ejemplo en México, específicamente en el estado de Puebla, la creación de los hospitales integrales existentes en los servicios de salud en las regiones prioritarias, que garanticen un área de influencia interestatal indígena. Al respecto y con base en Gutiérrez, Betancourt y Betancourt (2008), se puede agregar que:

Con la creación de la Coordinación Estatal de Hospitales Integrales con Medicina Tradicional en los Servicios de Salud del estado de Puebla nace en el 2002, el Programa de Hospitales Integrales con Medicina Tradicional (PHIMT) cuyo objetivo principal es la de proporcionar servicios mixtos de salud y disponer de espacios donde se desarrolle la medicina tradicional y la medicina alópata en un marco de interculturalidad (párr. 2).

Así, se constituyó un grupo técnico de trabajo integrado por funcionarios federales de Planeación y la Coordinación General del Plan Puebla Panamá en busca de optimizar la calidad en la salud:

El proyecto surgió a partir del reconocimiento del distanciamiento entre la medicina alópata y la población indígena, así como del respeto a la cultura y forma de organización de esta misma población, en virtud de que poseen un acervo de conocimientos y prácticas valiosas para proteger su salud (GUTIÉRREZ; BETANCOURT; BETANCOURT, 2008, párr. 4).

Entre sus objetivos centrales del modelo de atención buscó la conexión entre la medicina alópata y la medicina tradicional indígena, así como garantizar espacios apropiados, para el desarrollo de los dos modelos, con la finalidad de contribuir a mejorar las condiciones de salud y vida de la población indígena. Sin embargo, poco se ha podido logar debido al peso que juega el conocimiento científico. Por un lado, a la fecha, siguen siendo descalificadas las enfermedades no reconocidas por la ciencia como el susto, la tristeza, la tiricia, entre otras; así como aquellas vinculadas con las de la espiritualidad.

Por otro lado, los médicos y enfermeras en estos espacios de interacción frente a los 
curanderos y parteras tradicionales, terminan por callarlos e ignorarlos, desconociendo sus saberes y conocimientos ancestrales. Lo más lamentable se presenta cuando los y las profesionales de la salud provienen de comunidades indígenas, por lo que el tema de la interculturalidad resulta complejo y con serias fracturas y en muchos casos, como sucede también en la escuela, se torna con tintes de conflictos y tensiones culturales. Esto ha sido posible de estudiar gracias a los diversos proyectos de investigación que venimos desarrollando con estudiantes de licenciatura y de posgrado en el área de la salud en la BUAP.

Al respecto Alarcón, Vidal y Neira (2003) nos señalan que:

\begin{abstract}
Situaciones como rechazo y falta de adhesión a las prácticas médicas occidentales, conflictos de poder entre agentes tradicionales y médicos, desarticulación de la estructura social de la comunidad, son elementos frecuentemente descritos en la literatura de salud internacional, especialmente en países como los nuestros, con altos índices de población indígena (p. 1064).
\end{abstract}

En este contexto, el desafío para el sector salud en México para las próximas décadas será, no solamente el de crear modelos de salud que respondan a las expectativas materiales y culturales del usuario, en un proceso de diálogo e intercambio cultural entre medicinas sino el de transformar la manera en como estamos formando a nuestros profesionales de la salud, dando todo el peso a la ciencia y a la medicina alópata en lo particular.

\title{
La experiencia de la Educación Popular en Salud en Brasil
}

En contraparte, mencionaremos que en el caso de Brasil una propuesta viable referencial es el Programa de Educación Popular en Salud, que bajo el sistema de tiendas de afecto cubren gran parte de las necesidades de su población en estos sentidos.

La Educación Popular en Salud no es una medicina alternativa que surge por la inaccesibilidad a servicios apropiados de salud, que se adueña del "prueba y error" y de saberes populares, improvisando una respuesta a las necesidades que se detectan diariamente. La Educación Popular en Salud es el nuevo camino de asociación, solidaridad y cooperación entre los miembros de la comunidad.

Se plantea que la educación popular puede constituirse como un paradigma, y a la vez una propuesta metodológica, que ponga como centro de la acción el reconocimiento de la persona como un actor protagonista de su historia y sus prácticas, reconociendo sus saberes y cultura. "Es la que nos saca de la parálisis en la que nos han dejado tantos años de hegemonía médicohospitalaria" (VIVERO ARRIAGADA, 2016, p. 426).

Por tanto, la Educación Popular es una educación comprometida y participativa orientada por la perspectiva de todos los derechos de los pueblos. Su principal característica es utilizar el saber de la comunidad como 'materia prima' de la enseñanza. Es aprender a 
partir del conocimiento del sujeto y enseñar a partir de las palabras y temas generadores de su cotidiano. El proceso de enseñanza-aprendizaje es mirado como un acto de conocimiento y transformación social, llevando en cuenta la perspectiva política.

En Brasil fue creada a partir de una lucha de los movimientos sociales en conjunto con profesionales de salud e investigadores que impulsaron una Política Nacional de Educación en Salud Popular. Esta tiene principios metodológicos para orientar su ejecución en los cotidianos de salud. Los principios teóricos metodológicos de La Política Nacional de Educación en Salud Popular del Brasil (PNEPS-SUS, 2013) son:
a) El diálogo
b) El amor
c) La problematización
d) Construcción compartida de conocimiento
e) Emancipación
f) Compromiso con la construcción del proyecto Popular Democrático

Así la Educación Popular no se hace para el pueblo, sino con el pueblo. Tiene como punto de partida el proceso pedagógico del saber desarrollado en el trabajo, en la vida social y en la lucha por la sobrevivencia y busca incorporar las formas de sentir, pensar y actuar de los grupos populares, trayendo así, una referencia para hacer una gestión participativa en salud. O sea, es una manera de hacer salud, fundamentado tradicionalmente en las formas populares de cuidar, desarrollando posibilidades de construcción de procesos de cuidado dialogados, participativos y humanos, acogedores de la cultura y de los saberes populares.

\section{Las Tiendas de Afecto Popular (TAP)}

Una de las preguntas que siempre nos hemos planteado para el tema de la diversidad de sistemas en salud es la siguiente: ¿Cómo hacer salud con interculturalidad respetando los saberes ancestrales y tradicionales?

A partir de la Educación Popular en Salud es posible porque se trabaja con la horizontalidad/lateralidad. El cuidado del otro y la otra forma parte de la educación popular porque creemos que no hay cambios sin afecto, sin que las personas estén implicadas/dispuestas a estar con los otros para tener un diálogo. Y por lo tanto habrá significación. De esa forma una de las metodologías que usamos son las Tiendas de Afecto Popular (TAP), las cuales, cabe aclarar, fueron inspiradas en las propuestas conceptuales de Paulo Freire (1993), sobre la amorosidad en la enseñanza y el diálogo horizontal.

Se tratan de espacios no fijos, que circulan por diversos espacios, barrios y zonas dentro del interior de algunos estados de Brasil, es decir son desmontables. Son vistas como 
una estructura física, mediada por las emociones, donde se hacen "paseos en los excesos de certezas", conocimiento, saberes, poderes de todos los que participan en ese espacio y experiencia. Incorpora y recupera la apreciación de las personas, la inclusión no para normalizar las singularidades de la elaboración de círculos de cuidado, no siempre redondos, pero cada uno tiene la oportunidad de reconocer al otro en el espacio propio y del otro. Se producen a partir de la demanda de personas que participan en ella y lo identifican como un dispositivo de gran alcance para los problemas de salud y de la interculturalidad.

Esta experiencia se debe a sentimientos voluntarios, uno de tantos, la militancia por el derecho a la salud para todos, y se reconoce que en esta interfaz hay otros. Con las TAP este sentimiento se integra con otras personas. Eso hace que cada TAP sea diferente. En donde cada práctica de cuidado en atención a la salud enfermedad opere como un modo alternativo propio, singular. Por ello, recalcamos la importancia de las relaciones entre las culturas, sin restringirse a la cuestión de apenas comprender la cultura del otro y de la otra. Lo importante es comprender la relación entre las culturas y sistemas de salud. Por eso es imperativo no anteponer una cultura sobre otra (la occidental sobre las originarias). No se desconoce que transitar hacia estos modelos de salud intercultural, se presenten tensiones entre las culturas pero el punto es que se valoricen las relaciones interculturales que presuponen el diálogo horizontal, la compartición de saberes y conocimientos.

Sin embargo, instalados desde nuestros centros de trabajo tanto en México como en Brasil, se observan objetivos que no se cumplen, no sólo en cuestiones de misiones o visones institucionales, sino en el sentido de la inclusión cultural de sistemas de servicios de medicina, por un lado la occidental y por otra la que tanto ha aportado a la humanidad: el de la medicina tradicional. Esto deja al descubierto que muchas prácticas ancestrales y conocimientos milenarios son desaprovechados por la medicina y las instituciones acreditadas.

La necesidad de desarrollar procesos interculturales en educación y salud de países latinoamericanos por diversas razones históricas, sociopolíticas y epidemiológicas nos conducen a retomar las apreciaciones de Alarcón, Vidal y Neira (2003) sobre que la antropología como ciencia contribuye tanto a develar y comprender las culturas involucradas en el proceso de atención de salud, como a proporcionar elementos socioantropológicos, para apreciar el fenómeno de salud y enfermedad desde su dimensión biológica, social y cultural en un escenario de creciente pluralismo médico que caracteriza a la sociedad contemporánea. Es innegable que los conflictos entre diferentes sistemas médicos no emergen sólo de las diferencias en los modelos explicativos que los sustentan, sino también de la dominación social de un modelo de salud sobre otro, diría Jorge Gasché (2008), de esa relación de dominio y sumisión.

En este contexto, el desafío para los sectores de educación y salud en las próximas décadas será el de crear modelos y paradigmas aplicables a diálogos e intercambio cultural entre diversos sistemas de salud y de medicinas. De hecho, si se pretende mejorar la calidad de los cuidados a las personas se deben atender otras dimensiones tales como la 
espiritualidad y las emociones, para ello es necesario diseñar currículas; formar profesionales para ejercer una salud con enfoques transculturales e interdisciplinarios, con la finalidad de aprovechar conocimientos científicos y los conocimientos de culturas ancestrales lo cual repercutiría, indiscutiblemente, en modelos holísticos de alto impacto para la prevención de enfermedades y el ejercicio de una buena salud.

\section{Conclusiones}

Los estudios que hemos venido realizando en torno a temáticas de educación y salud intercultural en México y Brasil, evidencian en primera instancia algunos aspectos relevantes para revisar los mecanismos de articulación social para con los pueblos y poblaciones indígenas, así es importante discutir el papel que le toca jugar a la educación superior, por encima de políticas globalizantes, que asfixian y condicionan nuestros Estados nacionales pero sobre todo nuestro mundo actual: multicultural y plurilingüe.

El desarrollo de una política de educación y salud intercultural debe ser un tema de agenda internacional prioritario en los países latinoamericanos. En ese sentido, es conveniente abrir la posibilidad de reconocer los sistemas culturales y conocimientos ancestrales de nuestras poblaciones y vincularlos con actividades académicas que estén en el centro de interés, justamente es aquí en donde nuestras instituciones de educación superior tienen mucho que hacer por delante entre sus académicos y estudiantes, pero también con un compromiso social, sustantivo, entre las presentes y futuras generaciones.

Por otra parte, si se pretende mejorar la calidad de los cuidados a las personas se deben atender otras dimensiones tales como la espiritualidad y las emociones, para ello es necesario diseñar y ejercer una salud con enfoques transculturales e interdisciplinarios, con la finalidad de aprovechar conocimientos científicos y los conocimientos de culturas ancestrales lo cual repercutiría, indiscutiblemente, en modelos holísticos de alto impacto para la prevención de enfermedades y el ejercicio de una buena salud.

Políticas, intenciones, discursos y derechos, como vimos a lo largo del presente escrito existen, el reto que nos queda a todos los que conformamos estas sociedades multiculturales y plurilingües, indígenas y no indígenas, es cómo poner en marcha programas que centren la acción y la praxis de sus postulados en el reconocimiento ejercicio pleno cotidiano de sistemas diversos tanto en salud como en educación. Sólo de esa manera podemos garantizar paradigmas interculturales e inclusivos.

\section{Referencias}

ALCARCÓN, A.; VIDAL, A.; NEIRA, J. Salud intercultural: elementos para la construcción de sus bases conceptuales. Revista Médica de Chile, Chile, v. 131, p. 1061-1065, 2003. 


\section{https://doi.org/10.4067/S0034-98872003000900014}

CÁMARA DE DIPUTADOS DEL H. CONGRESO DE LA UNIÓN. Ley General de Derechos Lingüísticos de los Pueblos Indígenas. Diario Oficial de la Federación. México: última reforma 20 de junio de 2018.

CONSEJO ECONÓMICO Y SOCIAL DE LAS NACIONES UNIDAS. El derecho al disfrute del más alto nivel posible de salud: 11/08/2000. Ginebra: Comité de Derechos Económicos, Sociales y Culturales, 2000. https://doi.org/10.18356/6fd1da54-es

CORES, A. La educación popular en salud salva: proyecto freireano en tiempos del neoliberalismo. Cadernos Cedes, v. 29, n. 79, p. 419-427, 2009. https://doi.org/10.1590/S0101-32622009000300010

DIETZ, G.; MATEOS L. S. Interculturalidad y educación intercultural en México. Un análisis de los discursos nacionales e internacionales en su impacto en los modelos educativos mexicanos. México: SEP, 2011.

FAGETTI, A. Reflexiones en torno a la medicina tradicional. Comisión de los Derechos Indígenas: $2003 . \quad$ Disponible en: https://www.google.com/url?sa=t\&rct=i\&q=\&esrc=s\&source=web\&cd=1\&ved=2ahUKEwjg8e k7cflAhVEKaOKHX6OCO0QFjAAegQIARAC\&url=http\%3A\%2F\%2Fwww.cdi.gob.mx\%2Fpnud\% 2Fseminario 2003\%2Fcdi pnud antonella faguetti.pdf\&usg=AOvVawOrXR1Bfh6rEDrUUKeG dZsj Consultado: 31 oct.

FREIRE, P. Cartas a quien pretende enseñar. México: Siglo XXI Editores, 1993.

GASCHÉ, J. La motivación política de la educación intercultural indígena y sus exigencias pedagógicas ¿Hasta dónde abarca la interculturalidad? En M. Bertely, J. Gasché y R. Podestá (coords.). Educando en la diversidad. Investigaciones $\mathrm{y}$ experiencias educativas interculturales y bilingües. Quito-Ecuador: Ediciones Abya-Yala, 2008. p. 367-397

GUTIÉRREZ, M; BETANCOURT. Y.; BETANCOURT, I. Medicina tradicional indígena en Puebla: los hospitales integrales. Experiencias en la capacitación y transferencia tecnológica sobre plantas medicinales y preparados herbolarios. En Fitoterapia Mexicana. Medicina Tradicional Hospitales Integrales [Blog]. 5 de diciembre de 2008. Disponible en: http://medicinaindigena.blogspot.com/2008/ Consultado: 31 oct.

INSTITUTO NACIONAL DE ESTADÍSTICA GEOGRAFÍA E INFORMÁTICA. (2015). Encuesta intercensal. México: 2015. Disponible https://www.inegi.org.mx/programas/intercensal/2015/default.html Consultado: 31 oct.

INSTITUTO NACIONAL DE ESTADÍSTICA GEOGRAFÍA E INFORMÁTICA. // Conteo de Población y Vivienda. México: 2005. Disponible en: https://www.inegi.org.mx/programas/ccpv/2005/ Consultado: 31 oct.

MEDICINA TRADICIONAL INDIGENA EN PUEBLA: LOS HOSPITALES INTEGRALES MINISTERIO DA SAÚDE. Portaria no. 2.761, de 19 novembro de 2013. Disponible en: http://bvsms.saude.gov.br/bvs/saudelegis/gm/2013/prt2761 1911 2013.html Consultado: 31 oct.

MINISTROS DE SALUD DE LAS AMÉRICAS EN LA CIUDAD DE PANAMÁ. Agenda de salud para las Américas 2008-2017. Ciudad de Panamá: 2007. Disponible en: https://www.google.com/url?sa=t\&rct=i\&q=\&esrc=s\&source=web\&cd=1\&cad=rja\&uact=8\&v 
ed=2ahUKEwjapPam2sTIAhUKbKwKHf3KCEYQFjAAegQIAhAC\&url=http\%3A\%2F\%2Fwww1.pa ho.org\%2Fhq\%2Fdmdocuments\%2F2009\%2FAgenda Salud para las Americas 2008-

2017.pdf\&usg=AOvVaw0jgEOzl X4GMk7-x8R2kIr Consultado: 31 oct.

OFICINA DEL ALTO COMISIONADO DE LOS DERECHOS HUMANOS DE LAS NACIONES UNIDAS. Programa sectorial de salud 2007-2012. (2007). Recuperado 22 septiembre, 2019, de http://www. paho.org/hq/dmdocuments/2010/Politicas Nacionales Salud-Mexico-

Programa Sectorial de Salud 2007-2012.pdf

ORGANIZACIÓN DE LAS NACIONES UNIDAS PARA LA EDUCACIÓN, LA CIENCIA Y LA CULTURA. Conferencia Mundial sobre la Educación Superior-2009: La nueva dinámica de la educación superior y la investigación para el cambio social y el desarrollo.

ORGANIZACIÓN DE LAS NACIONES UNIDAS. Objetivos de desarrollo del milenio una mirada desde América Latina el Caribe. Chile: Naciones Unidas, 2005. Disponible en: https://repositorio.cepal.org/bitstream/handle/11362/2797/S2005002 es.pdf?sequence=1

\&isAllowed=y Consultado: 31 oct.

ORGANIZACIÓN DE LAS NACIONES UNIDAS. Objetivos de Desarrollo Sostenible. 2015. Disponible en: https://www.un.org/sustainabledevelopment/es/objetivos-de-desarrollosostenible/ Consultado: 31 oct.

ORGANIZACIÓN INTERNACIONAL DEL TRABAJO; OFICINA REGIONAL PARA AMÉRICA LATINA Y EL CARIBE. Convenio núm. 169 de la OIT sobre pueblos indígenas y tribales en países independientes. Declaración de las Naciones Unidas sobre los Derechos de los Pueblos Indígenas. Lima: 2014.

Recebido em: 31/10/2019.

Aceito em: 14/02/2020. 